\title{
Measurement of Collins Asymmetries in inclusive production of pion pairs in electron-positron collisions at BABAR
}

\author{
F. Anulli, on behalf of the BABAR Collaboration* \\ INFN, Sezione di Roma \\ E-mail: fabio.anulli@romal.infn.it
}

\begin{abstract}
We present measurements of Collins asymmetries [1] in the inclusive process $e^{+} e^{-} \rightarrow \pi^{+} \pi^{-} X$ at a center-of-mass energy of $10.54 \mathrm{GeV}$. We use a data sample of $45 \mathrm{fb}^{-1}$ collected by the BABAR experiment at the PEP-II B factory at SLAC, and consider pairs of charged pions produced in opposite hemispheres of hadronic events. We observe clear asymmetries in the distribution of the azimuthal angles of pion pairs in two distinct reference frames. The obtained results can be compared to previous measurements performed by the Belle experiment [2,3], and can be used for global analyses combining $e^{+} e^{-}$and SIDIS data, for the simultaneous extraction of the Collins fragmentation function and the transversity distribution function.
\end{abstract}

Sixth International Conference on Quarks and Nuclear Physics,

April 16-20, 2012

Ecole Polytechnique, Palaiseau, Paris

\footnotetext{
* Speaker.
} 


\section{Motivation for extraction of Collins function in $e^{+} e^{-}$annihilation}

Transverse spin-dependent effects in fragmentation processes were first proposed by Collins, who introduced the chiral-odd polarized fragmentation function $H_{1}^{\perp}\left(z, P_{h}^{\perp 2}\right)$ [1]. It describes the relation between the transverse spin of the fragmenting quark and the azimuthal distribution of the final-state hadrons around the quark momentum. Experimental evidence for non-zero Collins functions was found by the HERMES $[4,5]$ and COMPASS $[6,7]$ collaborations, from the analysis of semi-inclusive deep inelastic scattering (SIDIS) of leptons off transversely polarized hydrogen and/or deuteron targets. The cross sections for these processes are proportional to the convolution of the Collins function and the chiral-odd transversity distribution function. In fact, using the factorization theorem, the SIDIS cross section is written as:

$$
\sigma^{e p \rightarrow e h X}=\sum_{q} D F \times \sigma(e q \rightarrow e q) \times H_{1}^{\perp},
$$

where $\sigma(e q \rightarrow e q)$ is the cross section for the electron-quark hard scattering and $D F$ is the unpolarized transversity distribution function. It is the least known among the three leading-twist parton distribution functions needed for a complete description of the momentum and spin distributions of the quarks inside the nucleon [8] . In order to extract the transversity function from Eq.(1.1) an independent measurement of $H_{1}^{\perp}$ is needed.

The measurement of the Collins function can be performed in the $e^{+} e^{-}$annihilation process $e^{+} e^{-} \rightarrow q \bar{q} \rightarrow h_{1} h_{2} X$, in which the two hadrons $h_{1}$ and $h_{2}$ are detected in opposite jets. A non zero value of the Collins function produces an asymmetry in the azimuthal distribution of the two hadrons around the fragmenting $q \bar{q}$ direction. The Collins asymmetry is conveniently measured in two different reference frames. In the first frame, called thrust reference frame or RF12, the azimuthal angles $\phi_{1}$ and $\phi_{2}$ shown in Fig.1(a) are calculated with respect to the thrust axis, that is the axis that maximizes the longitudinal momentum of the event. The differential cross section is:

$$
\begin{aligned}
\frac{d \sigma\left(e^{+} e^{-} \rightarrow h_{1} h_{2} X\right)}{d z_{1} d z_{2} d \cos \theta d \phi_{1} d \phi_{2}} & =\sum_{q, \bar{q}} \frac{3 \alpha^{2}}{Q^{2}} \frac{e_{q}^{2}}{4} z_{1}^{2} z_{2}^{2}\left[\left(1+\cos ^{2} \theta\right) D_{1}^{(0)}\left(z_{1}\right) \bar{D}_{1}^{(0)}\left(z_{2}\right)+\right. \\
& \left.+\sin ^{2}(\theta) \cos \left(\phi_{1}+\phi_{2}\right) H_{1}^{\perp,(1)}\left(z_{1}\right) \bar{H}_{1}^{\perp,(1)}\left(z_{2}\right)\right]
\end{aligned}
$$

In the second-hadron-momentum reference frame or RF0, $\phi_{0}$ is the azimuthal angle of one pion with respect to the momentum of the other pion, as shown in Fig.1(b), and the differential cross section is:

$$
\begin{aligned}
\frac{d \sigma\left(e^{+} e^{+} \rightarrow h_{1} h_{2} X\right)}{d z_{1} d z_{2} d^{2} \mathbf{q}_{T} d \cos \theta_{2} d \phi_{0}} & =\frac{3 \alpha^{2}}{Q^{2}} z_{1}^{2} z_{2}^{2}\left\{\frac{1+\cos ^{2} \theta_{2}}{4} \mathscr{F}\left[D_{1}\left(z_{1}\right) \bar{D}_{1}\left(z_{2}\right)\right]+\right. \\
& \left.+\frac{\sin ^{2} \theta_{2}}{4} \cos \left(2 \phi_{0}\right) \mathscr{F}\left[\left(2 \hat{\mathbf{h}} \cdot \hat{\mathbf{k}}_{T} \hat{\mathbf{h}} \cdot \hat{\mathbf{p}}_{T}-\hat{\mathbf{k}}_{T} \cdot \hat{\mathbf{p}}_{T}\right) \frac{H_{1}^{\perp}\left(z_{1}\right) \bar{H}_{1}^{\perp}\left(z_{2}\right)}{M_{1} M_{2}}\right]\right\} \text { (1.3) }
\end{aligned}
$$

In eqs. (1.2) and (1.3), $D_{1}$ is the unpolarized fragmentation function, $z_{1}\left(z_{2}\right)$ is the fractional energy of hadron $h_{1}\left(h_{2}\right), Q^{2}$ is the $e^{+} e^{-}$center-of-mass energy squared, and the angles are defined in Fig.1. In conclusion, the azimuthal asymmetries induced by the Collins function in $e^{+} e^{-}$annihilation are proportional to $H_{1}^{\perp}\left(z_{1}\right) \times \bar{H}_{1}^{\perp}\left(z_{2}\right)$. 


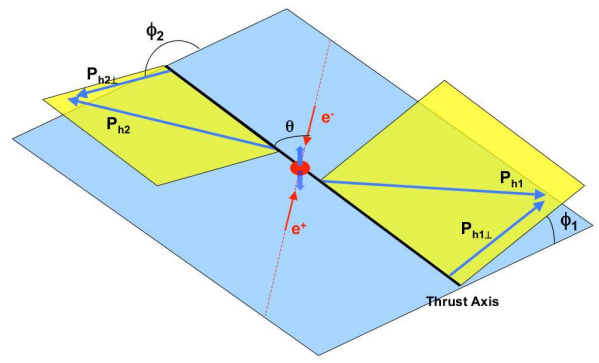

(a) RF12

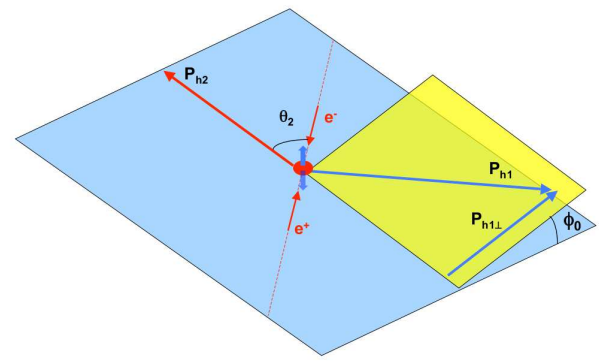

(b) RF0

Figure 1: (Color online) (a) RF12: $\theta=\theta_{T}$ is the angle between the beam and thrust axis; $\phi_{1,2}$ are the azimuthal angles between the scattering plane and the transverse momentum $P_{h \perp}$ around the thrust axis. (b) RF0: $\theta_{2}$ is the angle between the beam axis and the second hadron momentum; $\phi_{0}$ is the azimuthal angle between the plane spanned by the beam axis and the second hadron momentum $P_{2}$, and by the first hadron's transverse momentum $P_{1 \perp}$. All tracks are boosted in the $e^{+} e^{-}$center of mass frame.

\section{Analysis strategy}

The measurement of Collins Asymmetries is performed using a sample of data collected by the BABAR experiment at the center-of-mass energy of $10.54 \mathrm{GeV}$, that is $40 \mathrm{MeV}$ below the nominal energy of the collider, which corresponds to the peak of the $\Upsilon(4 S)$ resonance. For this reason, we refer to this data sample as the off-peak sample.

Assuming the thrust axis as $q \bar{q}$ direction and selecting pions in opposite hemispheres with respect to it, we measure the azimuthal angles $\phi_{1}, \phi_{2}$, and $\phi_{0}$. In order to select the two jets topology, we require a thrust higher than 0.8 . Only tracks identified as pions, consistent with being produced in the primary vertex of the event, and with a fractional energy $z=2 E / Q>0.2$ are considered. The total energy of the events is required to be higher than $7 \mathrm{GeV}$. The Collins asymmetries are obtained by measuring the $\cos (\phi)$ modulation of the normalized azimuthal distributions of the selected pion pairs, where $\phi=\phi_{1}+\phi_{2}$ or $\phi=2 \phi_{0}$ for the two reference frames. The asymmetries resulting by these distributions are largely affected by detector acceptance effects, making their measurement unreliable. We therefore perform suitable double ratios of the asymmetries in order to eliminate the detector effects and the first order of radiative corrections [9]. In particular we use the ratio of the normalized distributions of unlike charge $\left(R_{U L}\right)$ over those of like charge $\left(R_{L}\right)$ pion pairs. The double ratio can be written as:

$$
\frac{R^{U L}}{R^{L}}=\frac{N^{U L}(\phi) /<N^{U L}>}{N^{L}(\phi) /<N^{L}>}=\frac{1+\frac{\sin ^{2} \theta}{1+\cos ^{2} \theta} \cos (\phi) G^{U L}}{1+\frac{\sin ^{2} \theta}{1+\cos ^{2} \theta} \cos (\phi) G^{L}} \simeq 1+\frac{\sin ^{2} \theta}{1+\cos ^{2} \theta} \cos (\phi)\left\{G^{U L}-G^{L}\right\}
$$

$$
G^{U L}=\frac{\sum_{q} e_{q}^{2} \mathscr{F}\left(H_{1}^{f a v} H_{2}^{f a v}+H_{1}^{d i s} H_{2}^{d i s}\right)}{\sum_{q} e_{q}^{2}\left(D_{1}^{f a v} D_{2}^{f a v}+D_{1}^{d i s} D_{2}^{d i s}\right)}, G^{L}=\frac{\sum_{q} e_{q}^{2} \mathscr{F}\left(H_{1}^{f a v} H_{2}^{d i s}+H_{1}^{d i s} H_{2}^{f a v}\right)}{\sum_{q} e_{q}^{2}\left(D_{1}^{f a v} D_{2}^{d i s}+D_{1}^{d i s} D_{2}^{f a v}\right)}
$$


where $\theta$ is the polar angle of the thrust axis in the RF12 frame $\left(\theta=\theta_{T}\right)$ or the polar angle of the second hadron momentum in the RF0 frame $\left(\theta=\theta_{2}\right)$, as shown in Fig.1. Fitting the double ratio with a cosine function

$$
\frac{R_{U L}}{R_{L}}=B_{\alpha}+A_{\alpha} \cdot \cos (\phi),
$$

the parameter $A_{\alpha}$ contains just the Collins effect and higher order of radiative effects, since acceptance effects and radiative contributions do not depend on the charge combination of the pion pairs. Here $\alpha=12$ or $\alpha=0$ is used to distinguish the two reference frames.

\section{Study of systematic effects}

A crucial point for the measurement of Collins asymmetry is the identification of all the systematic effects that can influence the azimuthal distribution of the pion pairs.

Particularly significant is the dilution of the asymmetry due to the contamination of the selected pion pair sample by different background sources. The only two significant background processes are $e^{+} e^{-} \rightarrow c \bar{c}$ and $e^{+} e^{-} \rightarrow \tau^{+} \tau^{-}$. Their contribution to the measured asymmetry is estimated making use of both MC simulation and suitable data control samples, and then subtracted.

Another significant dilution of the asymmetry, affecting the measurement in RF12, is produced by the assumption of the thrust axis as the true $q \bar{q}$ direction, while this is only a rough approximation. An average correction factor of about 1.7 is estimated by using MC simulation.

\section{Preliminary results}

The preliminary results are presented in 10 symmetric combinations of $\left(z_{1}, z_{2}\right)$ intervals, as described in Tab.1. All significant systematic errors are evaluated and added in quadrature for each bin. In Fig.2 the BABAR preliminary results are compared with the superseded off-peak Belle data

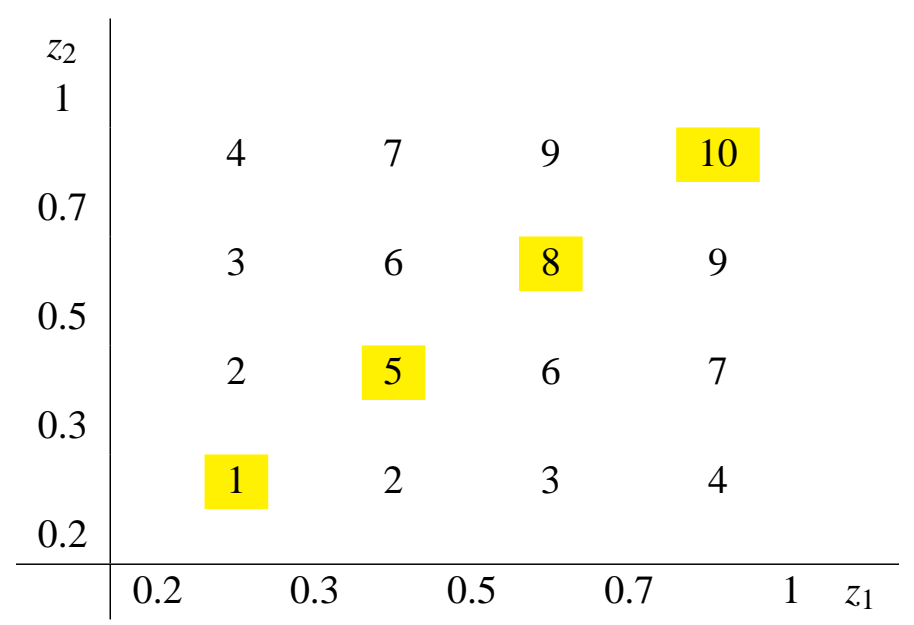

Table 1: Symmetrised $z$-bin subdivision and numbering scheme: the data sample is divided in 10 "bins" of $z_{1}$ and $z_{2}$. 

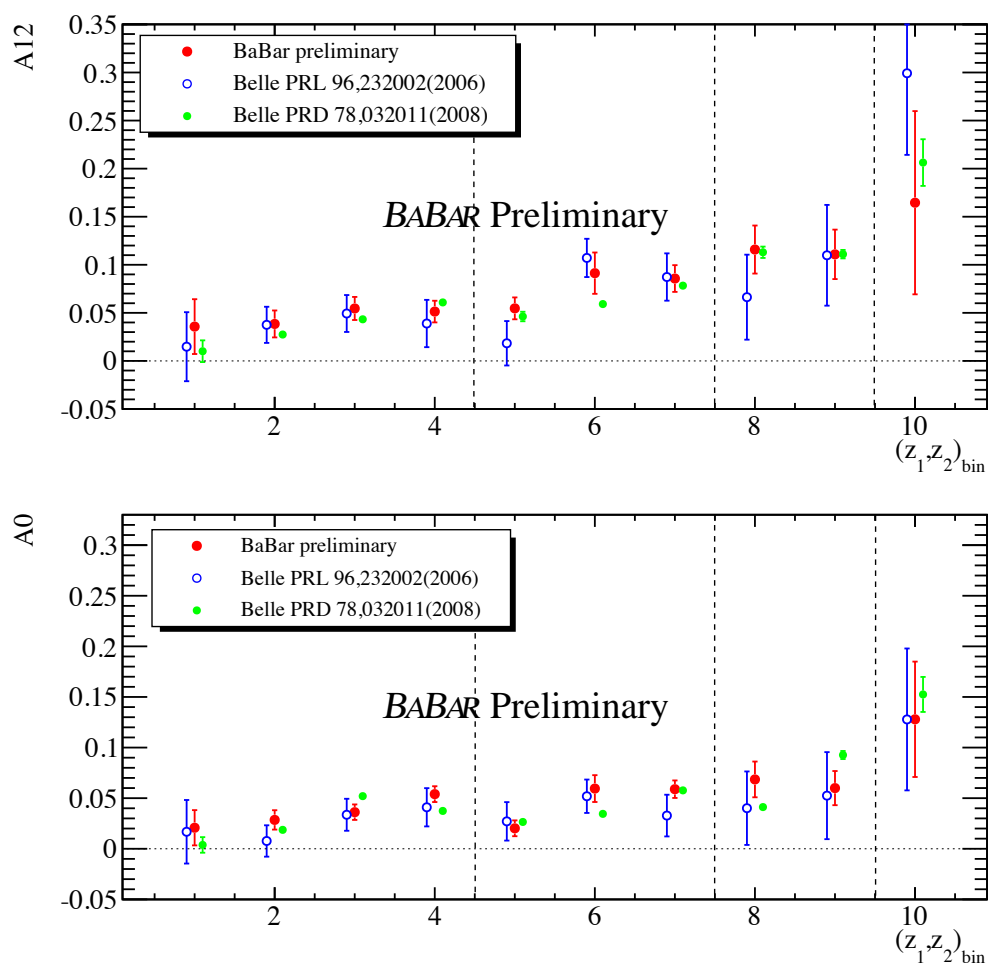

Figure 2: Preliminary BABAR measurement of Collins asymmetries (full circle in red), for RF12 (top) and RF0 (bottom). By comparison the superseded Belle off-peak results (open circle in blue), and Belle results on the full data sample (full green circles) are shown. Systematic and statistical errors are added in quadrature.

and the more recent combined off- and on-peak measurement obtained by the Belle Collaboration $[2,3]$. We should note that in the newest Belle publication [3], they estimate a new correction factor for the approximation of the $q \bar{q}$ axis with the thrust axis, so that we present the first Belle results on off-peak data corrected by the new factor for consistency. A significant increase of the asymmetries with increasing pion energies is observed, consistent with theoretical expectations.
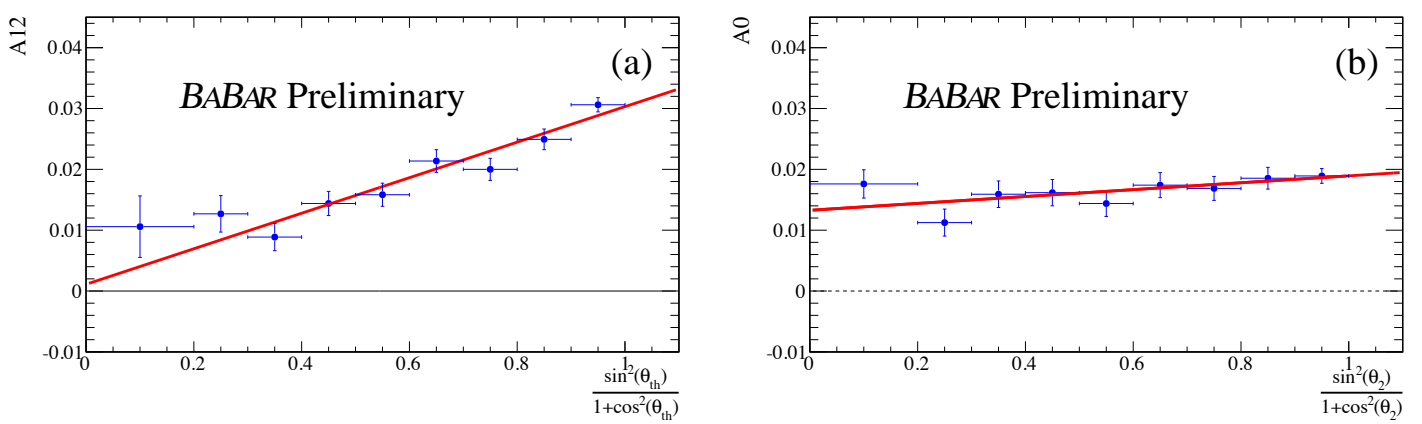

Figure 3: Collins asymmetry $A_{12}$ (a), and $A_{0}(\mathrm{~b})$, as a function of $\left(\sin ^{2} \theta\right) /\left(1+\cos ^{2} \theta\right)$, where $\theta=\theta_{T}$ and $\theta=\theta_{2}$ have been used in plot (a) and (b), respectively.

Following eq. (2.1), we studied also the dependence of the Collins asymmetries on $\sin ^{2} \theta /(1+$ 
$\left.\cos ^{2} \theta\right)$ in both reference frames, as reported in Fig.3. The expected linear dependence of asymmetry is observed in the thrust reference frame, but it seems not to hold in the second hadron momentum frame, where $\theta=\theta_{2}$. Such a behavior can be explained by the fact that the thrust axis describes the original $q \bar{q}$ direction better than the second hadron momentum.

\section{Conclusions}

We reported the preliminary $B A B A R$ measurement of Collins asymmetries in the pion system, performed using a data sample of about $45 \mathrm{fb}^{-1}$ collected at the energy of $10.54 \mathrm{GeV}$. The asymmetries are studied as a function of symmetric $\left(z_{1}, z_{2}\right)$-bins of the pion fractional energies and as a function of $\sin ^{2} \theta /\left(1+\cos ^{2} \theta\right)$, and are compared with the results published by the Belle Collaboration, finding an overall good agreement. The off-peak data sample is statistically limited, and we aim to publish a more complete set of measurements performed on the full BABAR data set.

\section{Acknowledgments}

We are very grateful to M. Anselmino, A. Bacchetta, M. Radici, and R. Seidl for the interesting discussions and the useful suggestions for performing this study. I also express many thanks to the Organizers for the warm hospitality and the very pleasant conference.

\section{References}

[1] J. C. Collins, Nucl. Phys. B396, (1993) 161, J. C. Collins, Phys. Lett. B536, (2002) 43.

[2] R. Seid et al. [Belle collaboration], Phys. Rev. Lett. 96 (2006) 232002.

[3] R. Seidl et al. [Belle collaboration], Phys. Rev. D78 (2008) 032011.

[4] A. Airapetian, et al. [HERMES Collaboration], Phys. Lett. B562, 182 (2003).

[5] A. Airapetian, et al. [HERMES Collaboration], Phys. Rev. Lett. 94, 012002 (2005).

[6] E. S. Ageev, et al. [COMPASS Collaboration], Nucl. Phys. B765, 31 (2007).

[7] M. Alekseev, et al. [COMPASS Collaboration], Nucl. Lett. B692, 240 (2010).

[8] M. Anselmino et al., Phys. Rev. D 75 (2007) 054032.

[9] D. Boer Nucl. Phys. B 806, (2009) 23-67. 